\title{
A Seamlessly Integrated Device of Micro- supercapacitor and Wireless Charging with Ultrahigh Energy Density and Capacitance
}

\section{Chang Gao}

Beijing Institute of Technology

Jiancheng Huang

Tianjin University

\section{Yukun Xiao}

Beijing Institute of Technology

\section{Guoqiang Zhang}

Beijing Institute of Technology

\section{Chunlong Dai}

Beijing Institute of Technology

\section{Zengling Li}

Beijing Institute of Technology

\section{Yang Zhao ( $\nabla$ yzhao@bit.edu.cn )}

Beijing Institute of Technology

\section{Lan Jiang}

Beijing Institute of Technology https://orcid.org/0000-0003-0488-1987

\section{Liangti Qu}

Tsinghua University

\section{Article}

Keywords: Movable Charging, Contactless Micro-electronics, Flexible Micro-robotics

Posted Date: October 21st, 2020

DOI: https://doi.org/10.21203/rs.3.rs-83552/v1

License: (c) (i) This work is licensed under a Creative Commons Attribution 4.0 International License. Read Full License

Version of Record: A version of this preprint was published at Nature Communications on May 11th, 2021. See the published version at https://doi.org/10.1038/s41467-021-22912-8. 



\section{A Seamlessly Integrated Device of Micro-supercapacitor and Wireless Charging with Ultrahigh Energy Density and Capacitance}

Chang $\mathrm{Gao}^{1}$, Jiancheng Huang², Yukun Xiao', Guoqiang Zhang ${ }^{1}$, Chunlong Dai $^{1}$, Zengling $\mathrm{Li}^{1}$, Yang Zhao ${ }^{1 *}$, Lan Jiang ${ }^{3}$, Liangti $\mathrm{Qu}^{1}{ }^{1 *}$

${ }^{1}$ Key Laboratory of Cluster Science, Ministry of Education of China, Beijing Key Laboratory of Photoelectronic/Electrophotonic Conversion Materials, School of Chemistry and Chemical Engineering, Beijing Institute of Technology, Beijing 100081, P. R. China

${ }^{2}$ School of Microelectronics, Tianjin University, Tianjin 300072, P. R. China

${ }^{3}$ Laser Micro-/Nano-Fabrication Laboratory, School of Mechanical Engineering, Beijing Institute of Technology, Beijing 100081, P.R. China

${ }^{4}$ Key Laboratory of Organic Optoelectronics \& Molecular Engineering of Ministry of Education, Department of Chemistry, Tsinghua University, Beijing 100084, P. R. China.

Corresponding author. E-mail address: yzhao@bit.edu.cn (Y. Zhao); lqu@mail.tsinghua.edu.cn (L. Qu)

Abstract: Microdevice integrating energy storage with wireless charging could provide new opportunities for electronics design, such as moveable charging. Herein, we report a seamlessly integrated wireless charging microsupercapacitors (IWC-MSCs) by taking advantage of a designed highly consistent material system that both wireless coil and electrode are of the graphite paper. The transferring power efficiency of the wireless charging is 52.8\%. Benefitting from unique circuit structure, the intact IWC-MSCs displays low resistance and excellent voltage tolerability with a capacitance of $454.1 \mathrm{mF}$ $\mathrm{cm}^{-2}$, superior to state-of-the-art conventional planar MSCs. Besides, a record 
high energy density of $567.6 \mu W h \mathrm{~cm}^{-2}$ exceeds the existing metal ion hybrid MSCs and even commercial thin film batteries $\left(350 \mu W h \mathrm{~cm}^{-2}\right)$. After charging for 6 minutes, the IWC-MSCs reaches up to a power output of $4.5 \mathrm{~mW}$, which can drive an electrical toy car immediately. The specific electrode layout brings new insight for contactless micro-electronics and flexible micro-robotics. 


\section{Introduction}

Miniaturized energy storage devices with flexibility and portability have become increasingly important in the development of next generation electronics. ${ }^{1-5}$ Generally, it still needs to find efficient connection ways for the energy storage microdevices and routine power supply equipment to complete the charging process, which runs counter to the original intention and future development of microdevices. To this end, replacing traditional electric supply mode with contactless charging can enhance the energy storage microdevices in micro-drones, micro-electric vehicles, and micro-detective system by eliminating the cumbersome circuit external connecting procedure.

Microdevices that combine energy storage and wireless charging functions can be defined as integrated wireless charging energy storage microdevices. These wireless charging microdevices have been explored as means to get rid of the complicated charging wires and foreseeable physical electrode contact damage of microelectronic equipment in the current research stage. It is known that the wireless charging capability strongly depends on electrical conductivity and structural of coils that receive the magnetic induction signal. Many natural metals and metal-based composites can generate high received current, but they are easy to oxidize and corrode, resulting in shortened lifetime. ${ }^{6-8}$ Furthermore, their high heat loss will consume a large amount of electric energy, reducing the practical utilization. Early studies on the wireless charging microdevices have also shown high energy consumption of joints and the 
vulnerable structural of the integrated microdevices due to the crude assembly of metal coils and electrodes. In theory, carbon materials with low cost, good stability, corrosion-resistant, and high conductivity may achieve similar performance to metal coils while overcoming their shortcomings. ${ }^{9}$ However, the engineering realization of a high-performance metal-free coil in wireless charging microdevices is hindered by the existing material systems due to the dearth of effective structural design and assembly protocols.

Micro-supercapacitors (MSCs) are particularly attractive in wireless charging storage microdevices because of their fast charging and discharging rate (adapting to changeable voltage), high power density (large driving force) and splendid cycling stability. ${ }^{10-14}$ However, current versions of wireless charging MSCs are difficult to implement as the wholeness devices in further practical applications because of the lack of suitable material engineering compatibility under the existing wireless charging coil mode. ${ }^{15,16}$ Except for the reductions of integrity and flexibility, the energy output is also prone to being affected, which is not to mention acquiring possible breakthroughs in highcapacity of wireless charging microdevices. ${ }^{17,18}$

To address these challenges, we herein propose seamlessly integrated wireless charging MSCs (IWC-MSCs) by taking advantage of a designed highly consistent material system that wireless coils and electrodes are of the same material origin. Graphite paper (GP) with rapid electron transfer capability, splendid corrosion resistance and wide operating voltage, is a satisfactory 
candidate material for both coils and MSCs electrodes in this system. ${ }^{19}$ Combined the integrated circuit design, the IWC-MSCs could reach an excellent intact structure, low current drain and excellent voltage tolerability. In addition, the resulting IWC-MSCs with a remarkably high areal capacitance of $454.1 \mathrm{mF} \mathrm{cm}^{-2}$ is superior to the conventional planar MSCs reported previously. Apart from the wide potential range of $3 \mathrm{~V}$, the IWC-MSCs device displays a record high energy density of $567.6 \mu \mathrm{Wh} \mathrm{cm}-2$, even larger than the commercial thin film batteries $\left(350 \mu \mathrm{Wh} \mathrm{cm}{ }^{-2}\right) \cdot{ }^{20}$ Moreover, the IWC-MSCs could drive an electrical toy car with wireless charging in only 6 minutes, indicating the great potential in practical applications.

\section{Results}

Design and fabrication of IWC-MSCs. The IWC-MSCs device is consisted of two modules: MSCs and wireless charging coil (WCC) acting as antenna (Fig. 1a). To integrate the two parts, we propose a planar coaxial structure, in which the MSCs are in the center surrounded by a coil. A shared electrode (purple line in Fig. 1a) is designed to connect the MSCs and WCC. Interestingly, we find that the shared electrode can act as a conductive line of the coil to assist WCC to harvest energy from the magnetic field, while it is also an electrode of three interdigital parallel MSCs (blue lines in Fig. 1a). Here, the number of MSCs is three instead of filling the center of IWC-MSCs. This is because that the MSCs in IWC-MSCs are inevitably affected by the magnetic field in current system. 
According to Faraday's law of Electromagnetic induction, if all the four sides of a square (blue dotted line in Fig. 1a) are used as electrodes of MSC, a new closed coil will be generated, which causes a similar induced magnetic field as the peripheral WCCs. It will affect the potential difference of the electrodes and thus make an impact on the charge storage of the MSCs. As a result, the designed pattern of IWC-MSCs not only enables MSCs with the ability of wireless charging, but also efficiently integrates WCC and MSCs in one device with robust and intact structure.

In conventional wireless charging coils, the resistance of the coil is generally required to be lower than $1 \Omega /$ m during transport of significant current in actual antenna. ${ }^{9}$ However, most of the existing WCCs are limited to metalbased materials, which are easily corroded under harsh chemical conditions. ${ }^{6-}$ ${ }^{8}$ To ensure the high integrity of IWC-MSCs, there is a great need to explore the materials that can be used for both electrodes and coils. GP has been widely used as circuit or electrode material due to its low sheet resistance $(0.015 \Omega / \mathrm{m})$, high conductivity, splendid corrosion resistance, and high operating voltage ( $\sim 3$ V). To this end, we propose that the GP is an ideal candidate for both WCC and electrode current collector of MSC. ${ }^{19}$

Figure $1 \mathrm{~b}$ demonstrates the fabricating process of the integrate device. A commercial graphite paper $(\sim 50 \mu \mathrm{m})$ was used as the supporting and conductive substrate for MSC electrodes. The activated carbon (AC) mixed with poly(vinylidene fluoride)(PVDF), Ketjen black and N-methyl pyrrolidone (NMP) 
that forming electrode slurry were then homogeneously painted on the graphite paper with a planar brush to concisely control the thickness of the electrode. Here, PVDF, Ketjen black, and N-methyl pyrrolidone (NMP) were used as the binder, conductive particles, and solvent, respectively. After drying for $24 \mathrm{~h}$ in vacuum at $60^{\circ} \mathrm{C}$, the graphite paper coated with a layer of AC (GP-AC) was obtained. Thereafter, an integrated device with an organized graphic was ingeniously designed and manufactured by a simple one-step laser etching process on the GP-AC substrate. Finally, the patterned integrated device was transferred onto a transparent PET tape to make sure the device is robust and steady. As shown in Fig. 1c, the integrated IWC-MSCs device consists three interdigital MSCs connected in parallel (center of the chip) and wireless charging coil (outside of the chip) with a certain number of turns, in which the numbers of interdigital electrodes and coils are easily controlled by the laser etching machine in demands. Interestingly, the coil of the IWC-MSCs is constructed by extending one of the electrodes in MSCs to be as the antenna of wireless charging. This designed pattern subtly enables MSCs with the ability of wireless charging from magnetic field, and efficiently shortens the connecting distance of different components, generating a very low resistance of the whole device. Unlike the commercial cylindrical power source (such as AA battery), the integrated device with planarity feature presents good flexibility and maintains stable electrochemical properties under bending state (Supplementary Fig.1), showing great potential in portable and ultrathin 
electronics (Fig. 1d and 1e). The scanning electron microscope (SEM) image of interdigital electrodes of MSCs is shown in Supplementary Fig. 2, where a homogeneous AC layer attaches firmly on GP, and the electrodes have clear and smooth edge, indicating a total etch of laser writing. Both the gap and the width of the electrodes are $400 \mu \mathrm{m}$.

a

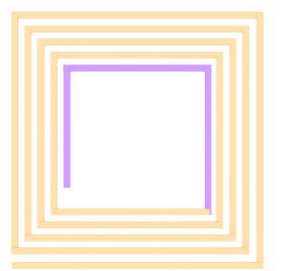

Wireless charging coil

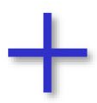

b

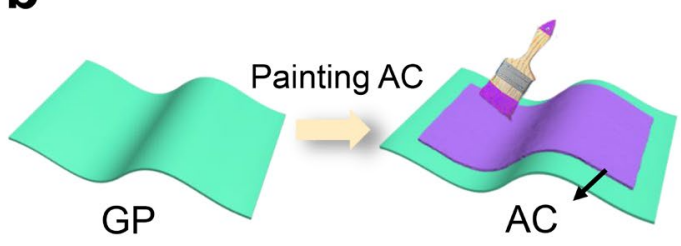

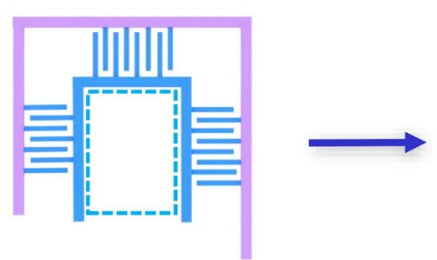

MSCs

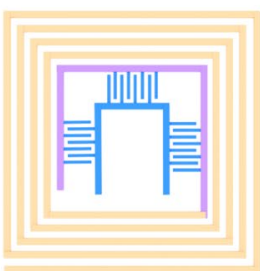

Integrated wireless charging MSCs (IWC-MSCs)
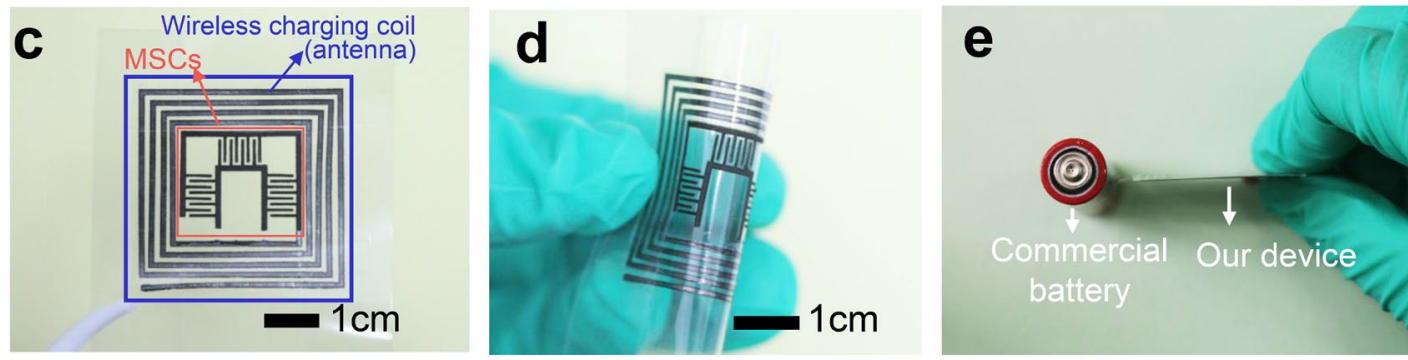

Fig. 1 The design, fabrication and electronic photos of IWC-MSCs. a) The combining of WCC and MSCs in the integrated device, IWC-MSCs. b) The schematic illustration of fabricating the integrated device. c) The image of the integrated device IWC-MSCs. d) Flexibility demonstration of the integrated device IWC-MSCs at a highly folded state. e) Thickness comparison of the commercial battery with IWC-MSCs. 
Electrochemical characteristics of MSC. The electrochemical characterizations of a single MSC unit was investigated to evaluate its electrochemical capabilities (Fig. 2a). The ionic liquid of 1-ethyl-3methylimidazolium bis (trifluoromethylsulfonimide) ([EMIM][TFSI]) with an excellent molar conductivity $\left(2.3 \mathrm{~cm}^{2} / \Omega \mathrm{mol}\right)$ and wide electrochemical window $(\sim 4.0 \mathrm{~V})$ was selected as electrolyte. ${ }^{21-22}$ Besides, the [EMIM][TFSI] could also provide high dielectric constant in double-layer capacitance of AC electrodes, contributing to store charges at the interface. ${ }^{23}$ In order to avoid the impact of the air factor and leakage of liquid electrolyte, the MSC was sealed with PET tape. As shown in Fig. 2b, the cyclic voltammetry (CV) curves of the MSC with different scanning rates of $20-100 \mathrm{mV} / \mathrm{s}$ present nearly rectangular shapes, indicating a good charge transport in MSC. ${ }^{1,24}$ Subtle redox peak may ascribe to a small part of the insertion of [EMIM] $]^{+}$into graphite layers at approximately $2.2 \mathrm{~V} .{ }^{25}$ The corresponding curves of galvanostatic charging/discharging are demonstrated in Fig. 2c, in which the shapes are near triangle and the IR drop is lower than $8 \%$ of the whole potential window, indicating domain ideal electrical double layer capacitance behaviors and low resistance. ${ }^{26}$ The inert resistance of the device is as low as $96 \Omega$ (Supplementary Fig.3). ${ }^{27}$

For further application of the MSC in practical, we connected the MSCs in parallel and series. It can be observed that the charging and discharging time of the curves of parallel device is three times of a single MSC (Fig. 2d), and the area of the enclosed curves of the parallel is almost three times of the single 
MSC (Fig. 2e). Besides, the series connected MSCs exhibits three times of potential window in a single MSC (Fig. $2 \mathrm{f}$ and $2 \mathrm{~g}$ ), suggesting ideal tandem and parallel behavior of the fabricated MSCs. Fig. $2 \mathrm{~h}$ reveals the areal capacitance of a single MSC calculated according to the current density. At a current density of $0.5 \mathrm{~mA} \mathrm{~cm}^{-2}$, the areal capacitance has a high areal capacitance of $63 \mathrm{mF}$ $\mathrm{cm}^{-2}$. Even if the current density increases to $2 \mathrm{~mA} \mathrm{~cm}^{-2}$, the areal capacitance reaches $35.3 \mathrm{mF} \mathrm{cm}^{-2}$, indicating excellent rate performance of the MSC. In accordance with the areal capacitance, the power density and energy density were obtained (Fig. 2i). When the power density is $0.75 \mathrm{~mW} \mathrm{~cm}^{-2}$ and $3 \mathrm{~mW}$ $\mathrm{cm}^{-2}$, the energy density is $78.7 \mu \mathrm{Wh} \mathrm{cm}{ }^{-2}$ and $44.2 \mu \mathrm{Wh} \mathrm{cm} \mathrm{cm}^{-2}$ respectively.

a
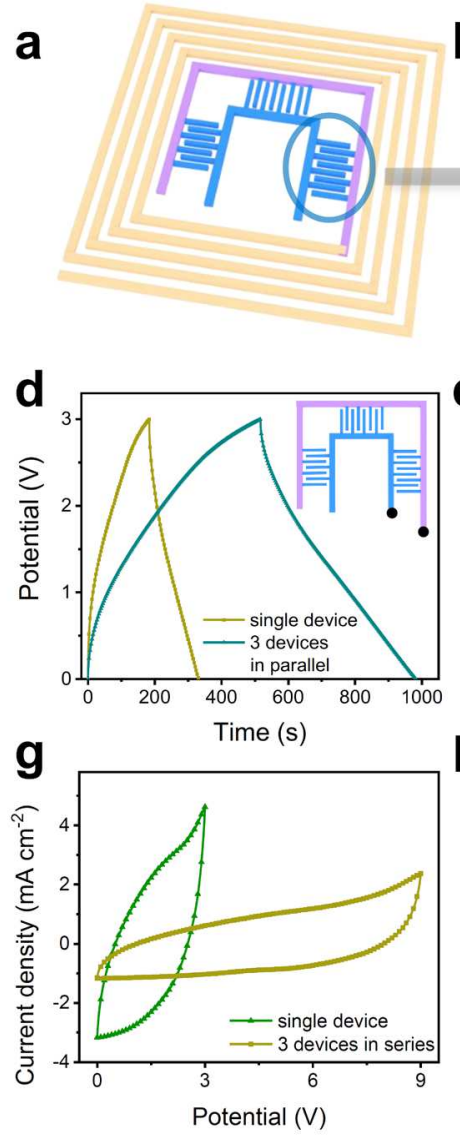

b

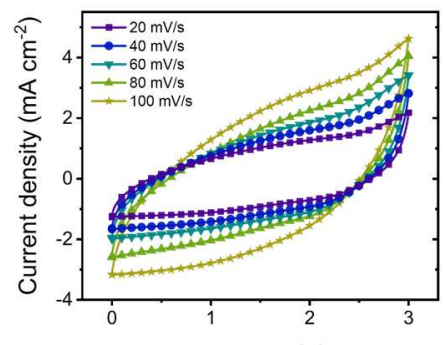

e

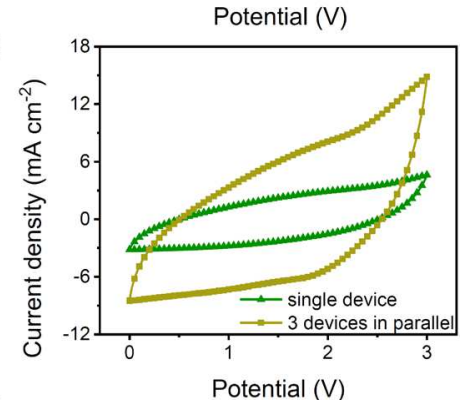

h

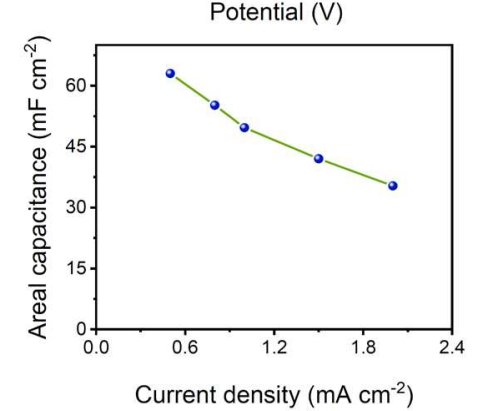

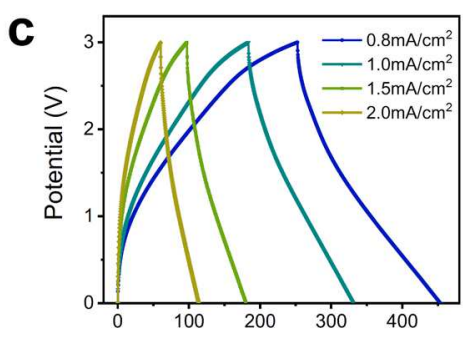

$f$

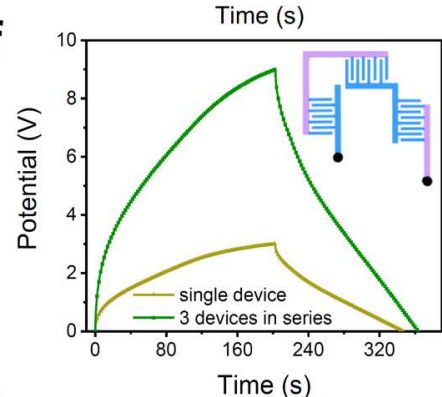

i

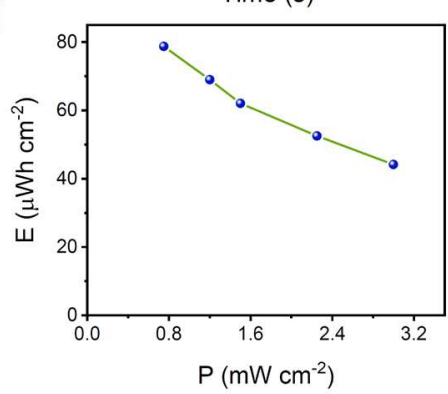

Fig. 2 Electrochemical properties of a single MSC in the integrated device. a) 
The scheme of one MSC in the integrated device. b) CV curves measured at scan rates of $20-100 \mathrm{mV} \mathrm{s}^{-1}$. c) The galvanostatic charging/discharging curves of a single MSC with different current densities. d) Galvanostatic charging/discharging curves of a group of three MSCs connected in parallel. The inset is the schematic diagram of parallel connecting details, where black dots are testing points. e) CV curves of MSCs connected in parallel. f) Galvanostatic charging/discharging curves of a group of three MSCs connected in series. The inset is the schematic diagram of serial connecting details, and the black dots are testing points. g) CV curves of MSCs connected in series. h) The specific capacitance of a single MSC calculated from the discharging time under different currents i) Power and energy densities of a single MSC unit.

Structural optimization and characterization of MSCs. For the practical use, there is a great need to increase the capacitance in one-unit device to satisfy the demand of high-power electronics. An IWC-MSCs device consists of three parallel MSCs, which is viewed as an integral device for measurement. To this end, we found that the capacitance of the integrated MSC could be further improved through simply doubling AC electrodes layer on both sides of the GP substrate without additional redundant devices (Fig. 3a and b). As shown in Fig. 3a, the structure of integrated MSCs device changes from a single layer to double layers after introducing extra AC electrodes on the other side of GP. The corresponding devices are named as single layered MSCs (SMSCs) and double layered MSCs (DMSCs), respectively. Fig. 3b reveals the crossing 
section diagrams of IWC-SMSCs (i) and IWC-DMSCs (ii). The single layer of AC material on the GP substrate possesses a thickness of $\sim 50 \mu \mathrm{m}$ (Supplementary Fig. 4a). While, the double layer shows a similar thickness of $\sim 50 \mu \mathrm{m}$ for each side of the GP substrate (Supplementary Fig. 4b). All the AC layers are in close contact with GP substrate. Moreover, the capacitance capability of the double layered integrated MSCs can be further optimized by increasing their numbers of the interdigital (blue color in Fig. 3a) without changing structure and shape of the device, and the improved MSCs are named as IMSCs.

Electrochemical performance of the overall IMSCs device was evaluated. As shown in Supplementary Fig. 5a, the CV curve area of the IMSCs device increases with the increase of the scan rates from 40 to $200 \mathrm{mV} / \mathrm{s}$, which is almost 2 times of the enclosed area for the SMSCs (Fig. 3c), indicating the strong electrochemical storage ability. According to the galvanostatic charging/discharging curves under various current densities, a series areal capacitance of IMSCs were calculated (Fig. 3d and Supplementary Fig. 5b). As revealed by Fig. 3d, the IMSCs exhibit an obvious areal capacitance advantage over the full range current densities than SMSCs. The areal capacitance of the IMSCs based on carbon material electrodes reaches an ultrahigh and maximum value of $454.1 \mathrm{mF} \mathrm{cm}{ }^{-2}$ at the current density of $1.6 \mathrm{~mA} \mathrm{~cm}-2$, far superior to those micro-supercapacitors reported previously (Supplementary Table 1). Remarkably, when the current is at $3 \mathrm{~mA}$ (Supplementary Fig. 5b), 
the discharging time is 146 seconds, revealing a large releasing current and long powering time of MSC. In addition, the IMSCs display an outstanding electrochemical process, which remain $95 \%$ of the original capacitance even after 2000 repeated cycles without any obvious declination (Supplementary Fig. 5c), indicating the excellent structural and electrochemical stability. These results demonstrate that the as-fabricated MSCs possess high bearable current to satisfy the current threshold of commercial electronics and drive a high energy demanding appliance.

Moreover, energy density and power density are crucial factors to evaluate the performance of energy storage device. As shown in Fig. 3e, the energy density of IMSCs are from $497.5 \mu \mathrm{Wh} \mathrm{cm} \mathrm{cm}^{-2}$ to $567.6 \mu \mathrm{Wh} \mathrm{cm}-2$ and power density are from $6.0 \mathrm{~mW} \mathrm{~cm}^{-2}$ to $2.4 \mathrm{~mW} \mathrm{~cm}^{-2}$ correspondingly. It is worth noting that the IMSCs display a supreme and the highest energy density among all planar micro-supercapacitors including hybrid MSCs, even commercial thin film battery $\left(350 \mu \mathrm{Wh} \mathrm{cm}^{-2}\right)$ (Fig. 3e) ${ }^{20,28-53}$. The mechanism for IMSCs with the high energy storage capacity is proposed as following:

(1) To investigate the electrochemical storage mechanism, the capacitive and diffusion-controlled contributions of the current were performed in Supplementary Fig. 6 . The result showed that a larger proportional capacitance was mainly contributed by electrostatic interactions between electrolyte ions and $A C$ particles. ${ }^{27}$ Besides, small parts of $[E M I M]^{+}$could also insert into graphite layers at $2.2 \mathrm{~V}$ (Fig. $2 \mathrm{~b})$ and provided an extra capacitance. This can 
be reflected by a small redox peak in the $\mathrm{CV}$ curves under fast charging and discharging process.

(2) The wide voltage window in this work is another important factor to achieve the high energy density. According to equation:

$$
E=0.5 C V^{2}
$$

where $C$ is the areal capacitance; $V$ is the potential range and $E$ is the energy density. It can be seen that the potential range plays an important role in energy density. Since the electrolyte of [EMIM][TFSI] could afford a brilliant voltage of $3 \mathrm{~V}$ in this system, the energy density was greatly improved based on the above equation.

(3) In traditional hybrid ion capacitors, the hybrid MSC usually contains one battery electrode to increase energy density and one supercapacitor electrode to improve power density, such as AC//graphite. ${ }^{54}$ The energy density demonstrates the storage of charges while the power density is like the import/export gate of the charges. Owing to the cross-section of MSC electrodes consisted with two layers (AC layer and graphite layer), the two layers possess equal chance to store/release charges during energy storage procedure. As a result, at the moment of releasing/storing, AC with large surface area plays a major role presenting a quite large power density, but with time going on, graphite featured high insertion space consequently also participates in the energy storage process therefore increases the energy density. It can be ascribed to the combination of $A C$ with graphite in this system that leading to a 
large energy density and capacitance. Furthermore, combination of $A C$ and graphite in one electrode would coincidentally balance the mass capacitance of two electrodes equally, which is regarded as the best proportional choice in hybrid device.

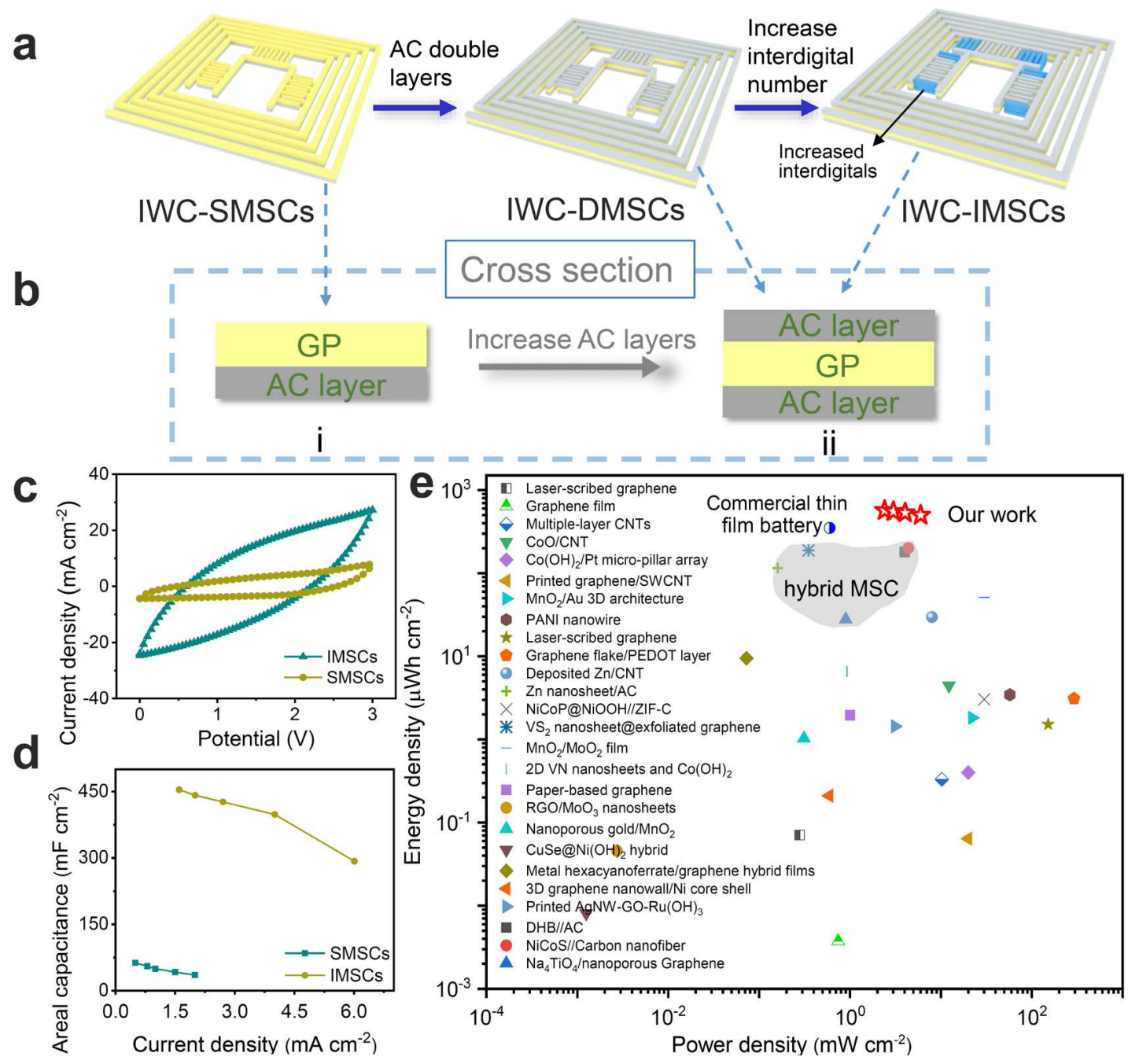

Fig. 3 Process and measurements of improving the performance of MSCs in device. a) Schematic diagrams of procedure of optimizing MSCs in IWC-MSCs. b) Change of cross section of SMSCs, DMSCs, and IMSCs. c) Comparison of CV curves under the scanning rate of $100 \mathrm{mV} / \mathrm{s}$ and d) rate capacity curves of MSCs of SMSCs and IMSCs, respectively. e) Ragone plots of the IMSCs 
compared with other reported MSCs.

Optimization of antenna of IWC-MSCs. As the antenna of IWC-MSCs, the charging coils enables MSCs with repetitive and sustainable charging capability without any additional electrical port. To optimize the collection of the electric energy from magnetic field, we investigated the main parameters of WCC as wireless charging part (Supplementary Fig. 7a), including gap of neighboring coils, and the distance between the receiving antenna and the transmitter. As displayed in Supplementary Fig. $7 \mathrm{~b}$, we find that when the gap between adjacent coils is $0.8 \mathrm{~mm}$, the receiving voltage is $\sim 5.8 \mathrm{~V}$, which is higher than the signal of 1.0 and $1.2 \mathrm{~mm}$ gap. It indicates that a narrow gap of neighboring coils is more efficient to the output of signals. On the other way, the distance between antenna and signal transmitter is another important factor of large signal reception. With the distance increasing from $0.5 \mathrm{~cm}$ to $1.0 \mathrm{~cm}$, the sensing voltage signal of the wireless charging part rises (Supplementary Fig. 7c). While it will fall back when continuing to increase the distance to 1.5 or 2.0 $\mathrm{cm}$ because of the changeable efficiency influenced by transmission distance. Consequently, we selected the optimal gap of $0.8 \mathrm{~mm}$ and transmission distance of $1.0 \mathrm{~cm}$ in this wireless charging system, leading to a high receiving current of $2.8 \mathrm{~mA}$ (Supplementary Fig. 7d). In addition, the receiving potential signals of the wireless charging part did not influence by the existence of the AC layer on WCC, showing the strong anti-interference ability (Supplementary Fig. 9). The inductance performance of antenna was also characterized by 
alternating current impedance method (Supplementary Fig. 10), which demonstrated good frequent stability of the charging coils as an antenna. Corresponding alternative current and voltage in transmitting coil of transmitter was detected by multimeter (Supplementary Fig. 8), which is $33.9 \mathrm{~mA}$ and $0.9 \mathrm{~V}$ respectively. The transferring power efficiency of the wireless charging is $52.8 \%$, indicating that the as-fabricated graphite WCC is a credible inductive antenna in this energy conversion system and the overall wireless charging system could be applied in practical applications.

Testing performance of the integrated IWC-MSCs. To evaluate the wireless charging performance of the integrate device, we further investigated the charging and discharging capability of the IWC-MSCs. Generally, a wireless charging system consists of transmitting part and receiving part as displayed in the circuit diagrams of Fig. $4 a$ and $4 b$. Here, the transmitting part mainly relies on a square coil made from square-shaped copper wires (Supplementary Fig. 11a), which can be charged by $10 \mathrm{~V}$ direct-current power source. As example, the IWC-SMSCs is used as the receiving part (the purple lines in Fig. 4b), which is connected with an external LED bulb to detect the discharging signal (Supplementary Fig. 11b). The wireless charging mechanism follows the principles of Electromagnetic Induction, leading to the conversion of from magnetic field energy to electrical energy. In the wireless charging process, the transmitting circuit delivered an alternating current in $\mathrm{L}_{1}$ (Fig. 4a) at first, causing a changeable magnetic field nearby. Then, the antenna ( $\mathrm{L}_{2}$ in Fig. $\left.4 \mathrm{~b}\right)$ detected 
the magnetic field and induced alternating magnetic flux and current in receiving part. To achieve the stable charging process, the rectifier diode $\left(D_{1}\right.$ in Fig. 4b) was applied to rectify alternating current into direct current. When connecting " $m$ " and "p" points (i), the wireless charging began (Fig. 4c). Substantial charges produced from WCC and then stored into the SMSCs of IWC-SMSCs. Details of energy receiving current and inductive charges of the IWC-SMSCs are shown in Supplementary Fig. 12. The sensed rectified current in the SMSCs gradually decreased (Supplementary Fig. 12a) and the induced electric charges were steadily accumulated with the charging time going on (Supplementary Fig. 12b), indicating that the magnetic field energy was transformed into electric energy by WCC and the electricity was reserved in SMSCs. In contrast, when switch "p" to "q" points (ii), the discharging process began (Fig. 4d). The charges stored in SMSCs were released and lit the red emitting diode $\left(D_{2}\right.$ in Fig. $\left.4 d\right)$. The entire wireless charging and detecting process is presented in Supplementary Movie 1. 


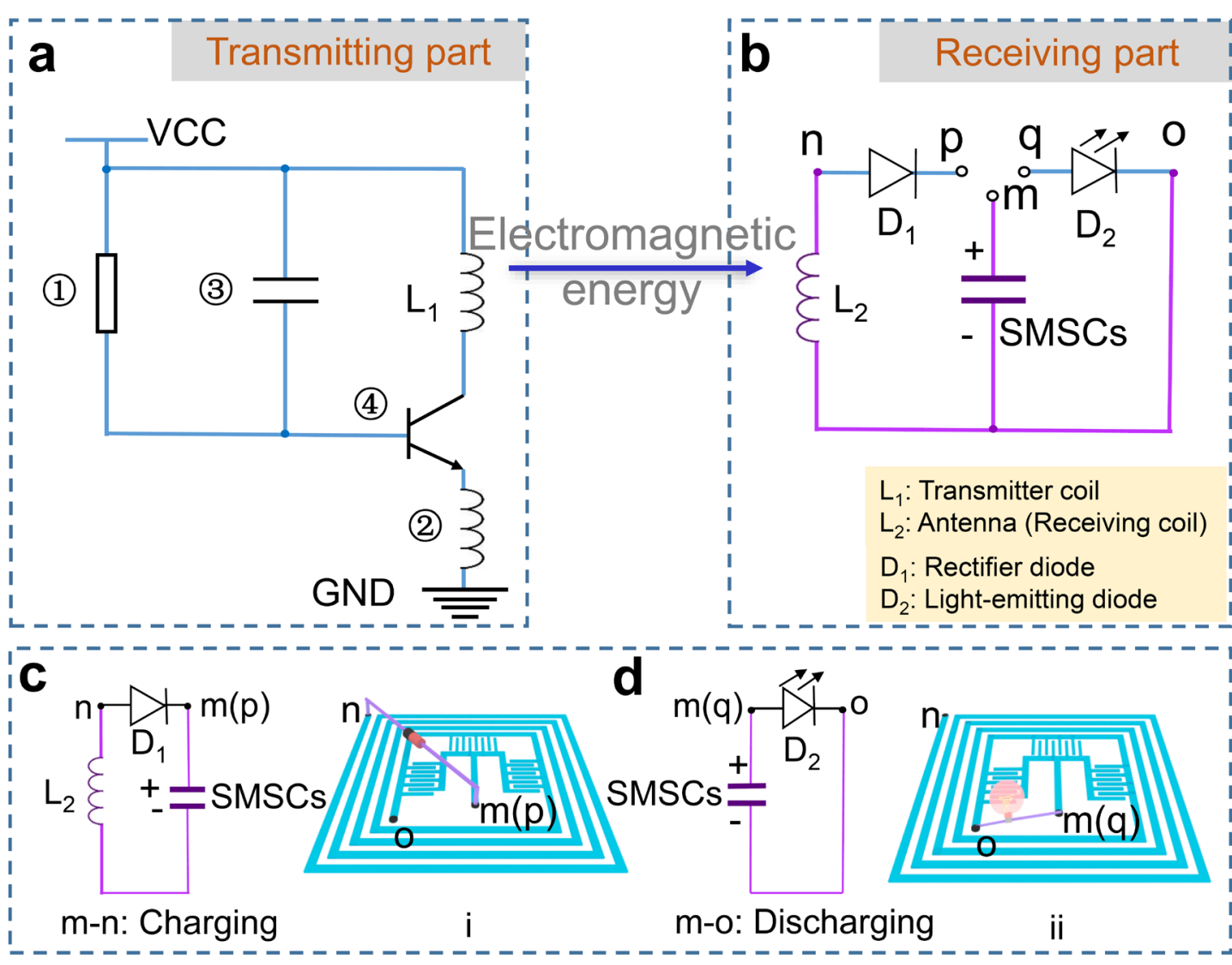

Fig. 4 Circuit diagram and working mechanism of wireless charging system. a)

Circuit diagram of wireless transmitting part and b) receiving part. c) Schematics of the circuit while connecting point "m" and "p" of IWC-SMSCs to charge SMSCs (i). d) Schematics of the circuit while connecting point " $m$ " and "q" of IWC-SMSCs to release the accumulated charges in SMSCs (ii), thus lighting a red LED. The IWC-SMSCs is in purple color in the circuit diagrams.

Application of IWC-IMSCs. The high-performance and integration of the wireless charging MSCs endow them with ability to be applied in practical mobile electronics. We found that an electrical toy car exhibited a starting current of $2.2 \mathrm{~mA}$, which was smaller than the discharge current $3 \mathrm{~mA}$ in Supplementary Fig. 5b. This indicated that the fabricated IMSCs could directly 
replace the commercial columnar battery (such as AA battery) to power an electrical car.

As present in Fig. 5a and 5b, copper foils are used as conducting wires to stick on the IWC-IMSCs, corresponding to $\mathrm{m}$, n, o points. Like the structure of circuit in Fig. 4b, the copper foil at " $n$ " point is connected with a rectifier diode, while the copper foil at "o" point is assembled with an electro-motor of the car. The wireless charging and discharging process is controlled by using a switch ("m" point) in the circuit diagram. Fig. $5 c$ and $5 d$ demonstrate the photograph of IWC-IMSCs on a commercial toy car (front and back). This integrated wireless charging energy storage device is easily attached on exterior of the car without complex fixing accessories, indicating the good environmental adaptability and operability. To complete wireless charging process, the car with IWC-IMSCs is used as the wireless receiver and placed nearby transmitter (Fig. 5e). When the switch is at "p" point, the IWC-IMSCs of the car is charged by antenna in magnetic field. After wireless charging for 6 minutes, the induced electric charges reach to $\sim 680 \mathrm{mC}$. Meanwhile, the whole energy and power output of the IWC-IMSCs achieve $0.28 \mathrm{mWh}$ and $4.5 \mathrm{~mW}$, respectively, which can drive an electrical toy car immediately once switching to "q" point (Fig. $5 f$ and Supplementary Movie 2).

The integration of wireless charging into MSCs greatly simplifies the practical charging process of the electronics and promotes the important applications such as moveable charging. 

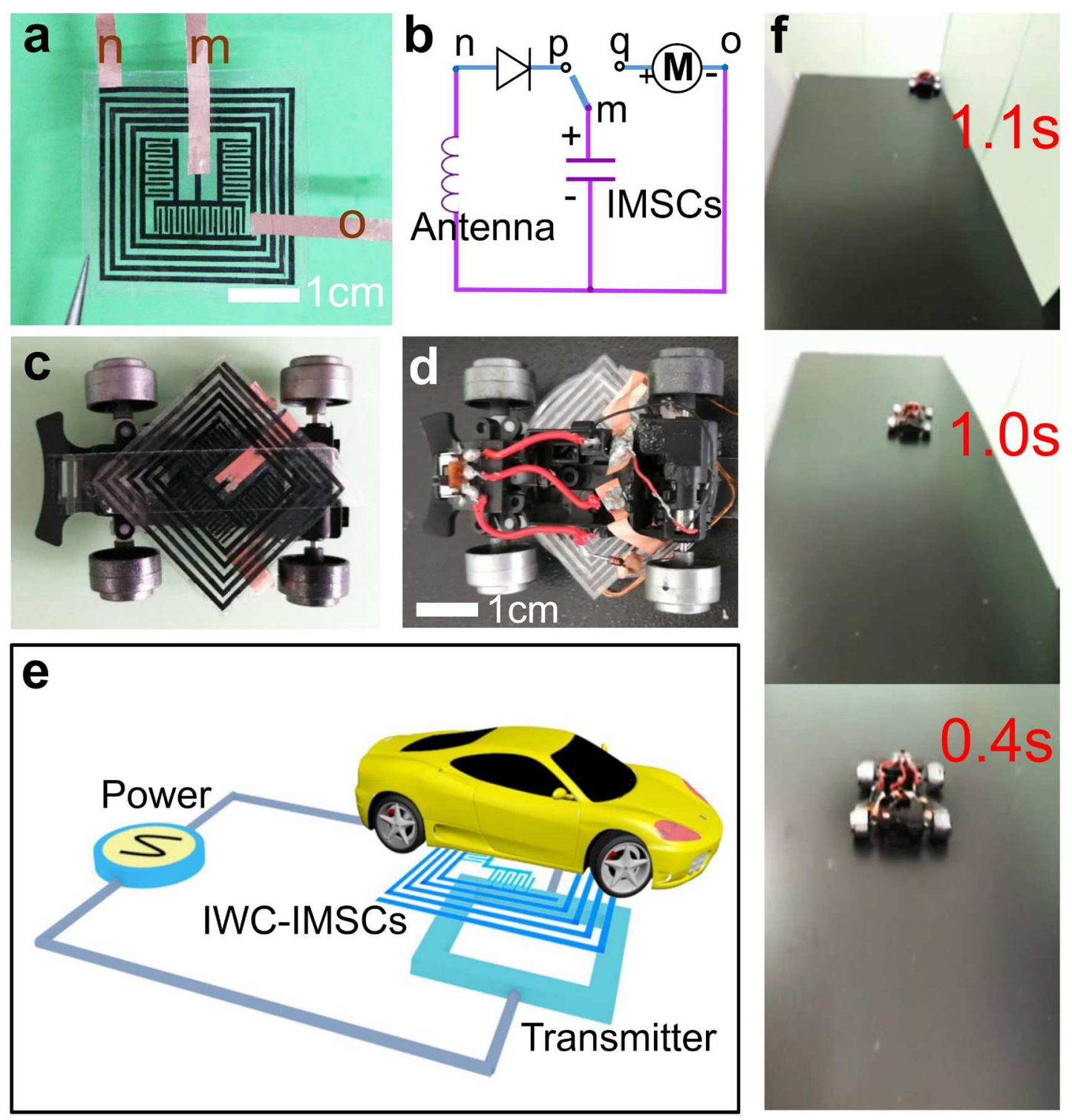

Fig. 5 Assembling IMC-IMSCs with an electrical toy car as a power source. a) An image of the connecting details of IWC-IMSCs using copper foils as conductors. "m", "n", "o" copper foils respectively correspond to "m", "n", "o" point in Fig. 5b. b) The circuit diagram of the IWC-IMSCs assembling with the car. c) The downside of the car assembled with IWC-IMSCs. d) The upside of the car connecting with a switch. e) The schematic diagram of the wireless charging system. f) The picture of the car with IWC-IMSCs running on the desk. 


\section{Discussion}

In conclusion, we developed an integrated and flexible wireless charging MSCs device by combining wireless charging coil and MSCs electrodes together through an ingenious designed pattern, which is for the convenience of contactless charging and rapid energy storage. This design enabled the IWCMSCs with a seamlessly contact structure and further with high conductivity and corrosion resistance properties by virtue of the oneness graphite material. Moreover, the as-prepared IMSCs of IWC-IMSCs possessed an ultrahigh areal capacitance (454.1 $\left.\mathrm{mF} \mathrm{cm}^{-2}\right)$ and a record high energy density $(567.6 \mu \mathrm{Wh} \mathrm{cm}$ ${ }^{2}$ ), superior to the reported planar MSCs. Furthermore, while assembling IWCIMSCs with an electrical toy car, the car could be driven immediately and ran quickly after only 6 minutes wireless charging. This work not only inspires new designs of integrated electrical device fabrication but also advances the convenience of miniature and portable electronics. 


\section{Methods}

Materials: Chemicals are from commercial sources with no further treatment.

Graphite paper (GP, $50 \mu \mathrm{m}$ thickness) and activated carbon (AC) specialized for supercapacitor are purchased from XFNANO Company of China. Polyvinylidene fluoride (PVDF) and N-methyl pyrrolidone (NMP) are analytical grade purchased from Alfa Aesar. Ketjen Black is used as conductive block (CB) and bought from Dodochem company in Suzhou city of China. 1-ethyl-3methylimidazolium bis (trifluoromethylsulfonimide) ([EMIM][TFSI]) is purchased from Innochem company in China. The toy car $\left(6.5 \times 5 \times 4 \mathrm{~cm}^{3}\right.$, mode number: 8024 ) is bought from Chengzhang toy company of Taobao shop in China.

\section{Preparation of micro-supercapacitor:}

(1) Preparation of IWC-MSCs pattern: 0.8g AC, 0.1g PVDF, 0.1g CB and 4mI NMP were added together, where AC was functioned as activated materials for capacitance and PVDF acted as binder. NMP was the solvent to dissolve all materials together. The mixture was ground for 20 minutes to obtain a homogeneous viscous slurry. Then, a planar bladder was used to coat the slurry on one side of graphite paper to make sure the surface smooth. After drying the $A C$ coated paper in vacuum oven for 24 hours at $60^{\circ} \mathrm{C}$, one $A C$ layer coated graphite paper was obtained. If there is further need, the other side of GP is coated with the same AC layer in the way. Double-layered GP was pressed by $5 \mathrm{MPa}$ pressure to make the GP-AC paper tight. The specific mass of each AC layer on SMSCs and IMSCs were $3.2 \pm 0.2 \mathrm{mg} / \mathrm{cm}^{2}$ and $11.9 \pm 0.5 \mathrm{mg} / \mathrm{cm}^{2}$ 
respectively for each side. To obtain interdigital electrodes and WCC pattern, we used laser machine $(20 \mathrm{~W})$ to scribe the as-prepared GP-AC paper. A total number of interdigital electrodes of SMSCs and IMSCs in IWC-MSCs were 24 and 39 , respectively. Besides, the gap of WCC changed with requirement (Supplementary Fig. 7b). After thorough ablation, the achieved pattern was transferred on PET tape, which was as the substrate of the device. The gap and the width of the interdigital electrodes in MSCs are all $400 \mu \mathrm{m}$.

(2) Assembly of IWC-MSCs: Copper foil was cut into stripes functioning as the conductive wires in the IWC-MSCs and stick with electrodes by conductive silver adhesives. To ensure no reaction related to the copper wire, each copper foil was well protected by PET tape to avoid contacting with graphite and electrolyte. Then, the electrolyte [EMIM][TFSI] was dropped on the electrode surface, and another PET tape was also used to encapsulate the entire device. Other junctions of the switch, wires and copper strips were soldered by melted tin.

Electrochemical measurements: All electrochemical tests were performed in a two-electrode system. Cyclic voltammetry (CV), galvanostatic chargedischarge tests, and electrochemical impedance spectroscopy (EIS) were carried out on a $\mathrm{CHI} 760 \mathrm{E}$ electrochemical workstation $(\mathrm{CH}$ Instruments Inc. Shanghai, China). The calculations of areal capacitance, energy density and power density are followed by our previous papers ${ }^{1}$, where the area of SMSCs and IMSCs are $0.3072 \mathrm{~cm}^{2}$ (approximately equals to $0.3 \mathrm{~cm}^{2}$ ) and $0.4992 \mathrm{~cm}^{2}$ 
(approximately equals to $0.5 \mathrm{~cm}^{2}$ ), respectively.

The areal capacitance $C_{a}$ was calculated as following:

$$
C_{a}\left(F \mathrm{~cm}^{-2}\right)=\frac{I(A) \times \Delta t(s)}{\Delta V(V) \times A\left(\mathrm{~cm}^{2}\right)}
$$

where $C_{\mathrm{a}}, I, \Delta t, \Delta V$, and $A$ are the areal capacitance $\left(\mathrm{F} \mathrm{cm}^{-2}\right)$, charge/discharge current $(\mathrm{A})$, discharge time $(\mathrm{s})$, discharge voltage $(\mathrm{V})$ and the area of the electrode $\left(\mathrm{cm}^{2}\right)$, respectively.

Calculation of energy density and power density:

$$
\begin{aligned}
& E\left(W h \mathrm{~cm}^{-2}\right)=\frac{C_{a}\left(F \mathrm{~cm}^{-2}\right) \times \Delta V^{2}(V)}{7200} \\
& P\left(W \mathrm{~cm}^{-2}\right)=\frac{E\left(W h \mathrm{~cm}^{-2}\right)}{\Delta t(\mathrm{~s})} \times 3600
\end{aligned}
$$

where $E$ and $P$ are the energy density $\left(\mathrm{Wh} \mathrm{cm}^{-2}\right)$ and power density $\left(\mathrm{W} \mathrm{cm}^{-2}\right)$, respectively.

The induced electric charges (Q) in IMSCs after 6 minutes charging was calculated by:

$$
\begin{gathered}
Q(m C)=C(m F) \times U(V) \\
C(m F)=C_{a}\left(m F \mathrm{~cm}^{-2}\right) \times A\left(\mathrm{~cm}^{2}\right)
\end{gathered}
$$

where $C$ is the capacitance of IMSCs; $C_{a}$ is $454.1 \mathrm{mF} \mathrm{cm}{ }^{-2}$ under the current density at $1.6 \mathrm{~mA} \mathrm{~cm}-2 ; A$ is the areal of IMSCs electrodes at $0.5 \mathrm{~cm}^{2} ; U$ is the voltage of the IMSCs at $3 \mathrm{~V}$.

The EIS tests were carried out in the frequency range of $10^{6}$ to $1 \mathrm{~Hz}$ with 5 $\mathrm{mV}$ of amplitude. Receiving rectified current signal of antenna are tested by Keithley $2612 \mathrm{~A}$ system sourcemeter while the $1 \mathrm{~N} 4148$ rectifier diode is to transverse alternative current into direct current. 
Characterization measurements: The scanning electron microscope (SEM) images was carried out on SUPRA 55. The laser source is LAJAMIN LASER (LM-YLP-20F-III). 


\section{References}

1 Gao, C. et al. Versatile origami micro-supercapacitors array as a wind energy harvester. J. Mater. Chem. A 6, 19750-19756 (2018).

2 Xue, Q. et al. Recent progress on flexible and wearable supercapacitors. Small 13, 1701827 (2017).

3 Gao, C. et al. A directly swallowable and ingestible micro-supercapacitor. J. Mater. Chem. 8, 4055-4061 (2020).

$4 \quad$ Lu, B. et al. Compact assembly and programmable integration of supercapacitors. Adv. Mater. 32, 1907005 (2020).

5 Gao, J. et al. Laser-assisted multiscale fabrication of configuration-editable supercapacitors with high energy density. ACS Nano 13, 7463-7470 (2019).

6 Kim, D. et al. Body-attachable and stretchable multisensors integrated with wirelessly rechargeable energy storage devices. Adv. Mater. 28, 748-756 (2016).

7 Wang, $X$. et al. Freestanding 3D mesostructures, functional devices, and shapeprogrammable systems based on mechanically induced assembly with shape memory polymers. Adv. Mater. 31, 1805615 (2019).

8 Araki, H. et al. Materials and device designs for an epidermal UV colorimetric dosimeter with near field communication capabilities. Adv. Funct. Mater. 27, 1604465 (2017).

9 Scidà, A. et al. Application of graphene-based flexible antennas in consumer electronic devices. Mater. Today 21, 223-230 (2018).

10 Sun, G. et al. A capacity recoverable zinc-ion micro-supercapacitor. Energy Environ. Sci. 11, 3367-3374 (2018).

11 Zhao, J., Li, Z., Zhang, M., Meng, A. \& Li, Q. Direct growth of ultrathin NiCo2O4/NiO nanosheets on $\mathrm{SiC}$ nanowires as a free-standing advanced electrode for highperformance asymmetric supercapacitors. ACS Sustainable Chem. Eng. 4, 3598-3608 (2016).

$12 \mathrm{Li}, \mathrm{B}$. et al. Electrode materials, electrolytes, and challenges in nonaqueous lithium-ion capacitors. Adv. Mater. 30, 1705670 (2018).

13 Wang, H., Zhu, C., Chao, D., Yan, Q. \& Fan, H. J. Nonaqueous hybrid lithium-ion and sodium-ion capacitors. Adv. Mater. 29, 1702093 (2017).

14 Gao, J. et al. Laser-assisted large-scale fabrication of all-solid-state asymmetrical micro-supercapacitor array. Small 14, 1801809 (2018).

15 Cai, J., Lv, C. \& Watanabe, A. Laser direct writing and selective metallization of metallic circuits for integrated wireless devices. ACS Appl. Mater. Interfaces 10, 915-924 (2017). Yue, Y. et al. A flexible integrated system containing a microsupercapacitor, a photodetector, and a wireless charging coil. ACS Nano 10, 11249-11257 (2016). 
coupled magnetic resonances. IEEE Trans. Ind. Electron. 58, 2906-2914 (2010).

Imura, T., Okabe, H. \& Hori, Y. in 2009 IEEE Vehicle Power and Propulsion Conference. 936-940 (IEEE).

19 Sivakkumar, S. R., Nerkar, J. \& Pandolfo, A. Rate capability of graphite materials as negative electrodes in lithium-ion capacitors. Electrochim. Acta 55, 3330-3335 (2010). Ferris, A., Garbarino, S., Guay, D. \& Pech, D. 3D RuO 2 microsupercapacitors with remarkable areal energy. Adv. Mater. 27, 6625-6629 (2015).

21 Fedorov, M. V. \& Kornyshev, A. A. Ionic liquids at electrified interfaces. Chem. Rev. 114, 2978-3036 (2014).

22 Galinski, M., Lewandowski, A. \& Stepniak, I. Ionic liquids as electrolytes. Electrochim. Acta 51, 5567-5580 (2006).

23 Lazzari, M., Mastragostino, M. \& Soavi, F. Capacitance response of carbons in solventfree ionic liquid electrolytes. Electrochem. Commun. 9, 1567-1572 (2007).

24 Shao, Y. et al. Design and mechanisms of asymmetric supercapacitors. Chem. Rev. 118, 9233-9280 (2018).

25 Huang, Y., Xiao, R., Ma, Z. \& Zhu, W. Developing dual-graphite batteries with pure 1ethyl-3-methylimidazolium trifluoromethanesulfonate ionic liquid as the electrolyte. ChemElectroChem 6, 4681-4688 (2019).

26 Shi, X., Zhou, F., Peng, J., Wu, Z. S. \& Bao, X. One-step scalable fabrication of graphene-integrated micro-supercapacitors with remarkable flexibility and exceptional performance uniformity. Adv. Funct. Mater. 29, 1902860 (2019).

27 Shao, Y. et al. Design and mechanisms of asymmetric supercapacitors. Chem. Rev. 118, 9233-9280 (2018).

$28 \mathrm{Li}, \mathrm{B}$. et al. Direct inkjet printing of aqueous inks to flexible all-solid-state graphene hybrid micro-supercapacitors. ACS Appl. Mater. Interfaces 11, 46044-46053 (2019).

29 Gong, J. et al. High-performance flexible in-plane micro-supercapacitors based on vertically aligned CuSe@Ni(OH $)_{2}$ hybrid nanosheet films. ACS Appl. Mater. Interfaces 10, 38341-38349 (2018).

$30 \mathrm{He}, \mathrm{Y}$. et al. Nano-sandwiched metal hexacyanoferrate/graphene hybrid thin films for in-plane asymmetric micro-supercapacitors with ultrahigh energy density. Mater. Horiz. 6, 1041-1049 (2019).

31 Zhang, P. et al. Zn-ion hybrid micro-supercapacitors with ultrahigh areal energy density and long-term durability. Adv. Mater. 31, 1806005 (2019).

32 Qiu, M., Sun, P., Cui, G., Tong, Y. \& Mai, W. A flexible microsupercapacitor with integral photocatalytic fuel cell for self-charging. ACS Nano 13, 8246-8255 (2019).

33 Gao, W. et al. Direct laser writing of micro-supercapacitors on hydrated graphite oxide films. Nat. Nanotechnol. 6, 496-500 (2011).

34 Shi, X. et al. Flexible, planar integratable and all-solid-state micro-supercapacitors 
based on nanoporous gold/ manganese oxide hybrid electrodes via template plasma etching method. J. Alloys Compd. 739, 979-986 (2018).

35 Zhang, P. et al. A nonaqueous Na-ion hybrid micro-supercapacitor with wide potential window and ultrahigh areal energy density. Batteries \& Supercaps 2, 918-923 (2019).

$36 \mathrm{Li}, \mathrm{J}$. \& Ono, T. in International Conference on Micro Electro Mechanical Systems. (IEEE).

37 Yadav, P., Basu, A., Suryawanshi, A., Game, O. \& Ogale, S. Highly stable laser-scribed flexible planar microsupercapacitor using mushroom derived carbon electrodes. Adv. Mater. Inter. 3, 1600057 (2016).

38 Wang, S. et al. All-solid-state high-energy planar hybrid micro-supercapacitors based on 2D VN nanosheets and $\mathrm{Co}(\mathrm{OH})_{2}$ nanoflowers. npj 2D Mater. Appl. 2, 7 (2018).

39 Nagar, B., Dubal, D. P., Pires, L., Merkoçi, A. \& Gómez-Romero, P. Design and fabrication of printed paper-based hybrid micro-supercapacitor by using graphene and redox-active electrolyte. ChemSusChem 11, 1849-1856 (2018).

$40 \mathrm{Li}, \mathrm{H} ., \mathrm{Liu}, \mathrm{S}$., Li, X., Wu, Z. \& Liang, J. Screen-printing fabrication of high volumetric energy density micro-supercapacitors based on high-resolution thixotropic-ternary hybrid interdigital micro-electrodes. Mater. Chem. Front. 3, 626-635 (2019).

41 Yu, W., Zhou, H., Li, B. Q. \& Ding, S. 3D printing of carbon nanotubes-based microsupercapacitors. ACS Appl. Mater. Interfaces 9, 4597-4604 (2017).

42 Zhu, Y. G., Wang, Y., Shi, Y., Wong, J. I. \& Yang, H. Y. CoO nanoflowers woven by CNT network for high energy density flexible micro-supercapacitor. Nano Energy 3, 46-54 (2014).

$43 \mathrm{Xu}, \mathrm{H}$. et al. Flexible asymmetric micro-supercapacitors based on $\mathrm{Bi}_{2} \mathrm{O}_{3}$ and $\mathrm{MnO}_{2}$ nanoflowers: larger areal mass promises higher energy density. Adv. Ener. Mater. 5, 1401882 (2015).

44 Tian, X. et al. Arbitrary shape engineerable spiral micropseudocapacitors with ultrahigh energy and power densities. Adv. Mater. 27, 7476-7482 (2015).

45 Bellani, S. et al. Scalable production of graphene inks via wet-jet milling exfoliation for screen-printed micro-supercapacitors. Adv. Funct. Mater. 29, 1807659 (2019).

$46 \mathrm{Li}$, Y. et al. Remarkable improvements in volumetric energy and power of $3 \mathrm{D} \mathrm{MnO}_{2}$ microsupercapacitors by tuning crystallographic structures. Adv. Funct. Mater. 26, 1830-1839 (2016).

47 Patil, S. J., Park, J. S. \& Lee, D. W. in International Conference on Micro Electro Mechanical Systems 1002-1005 (2019).

48 Wang, K. et al. An all-solid-state flexible micro-supercapacitor on a chip. Adv. Ener. Mater. 1, 1068-1072 (2011).

49 Elkady, M. F. \& Kaner, R. B. Scalable fabrication of high-power graphene microsupercapacitors for flexible and on-chip energy storage. Nat. Commun. 4, 1475 (2013). 
Lee, H. U. \& Kim, S. W. Pen lithography for flexible microsupercapacitors with layerby-layer assembled graphene flake/PEDOT nanocomposite electrodes. J. Mater. Chem. 5, 13581-13590 (2017).

51 Wang, N. et al. Boosting the capacitance of an aqueous zinc-ion hybrid energy storage device by using poly(3,3'-dihydroxybenzidine)-modified nanoporous carbon cathode. ACS Sustainable Chem. Eng. 7, 14195-14202 (2019).

52 Jiang, Q., Kurra, N., Xia, C. \& Alshareef, H. N. Hybrid microsupercapacitors with vertically scaled $3 \mathrm{D}$ current collectors fabricated using a simple cut-and-transfer strategy. Adv. Ener. Mater. 7, 1601257 (2017).

53 Zheng, S. et al. All-solid-state planar sodium-ion microcapacitors with multidirectional fast ion diffusion pathways. Adv. Sci. 6, 1902147 (2019).

54 Chen, G. Z. Supercapacitor and supercapattery as emerging electrochemical energy stores. Int. Mater. Rev. 62, 173-202 (2017).

\section{Acknowledgements}

This work was supported by the National Key R\&D Program of China (2017YFB1104300), NSFC (22075019, 21604003), Beijing Natural Science Foundation (2152028, 2164070), and Analysis \& Testing Center, Beijing Institute of Technology.

Author Contributions: C. G., Y. Z. and L. Q. contributed to the project design. Y. Z. and L. Q. elaborated the methodology and supervised the study. C. G. conceived and carried out the majority of experimental measurements. Y. X., G. Z., C. D. and Z. L. helped with the fabrication and characterization of materials and the devices. C. G. and J. H. analyzed the data. C. G., Y. Z. L. J. and L. Q. wrote the paper. All authors discussed the results and contributed to the paper.

\section{Additional information}

Supplementary Information accompanies this paper at http://www.nature.com/naturecommunications

Competing Interests statement: The authors declare no competing financial interests. 


\section{Figures}

a

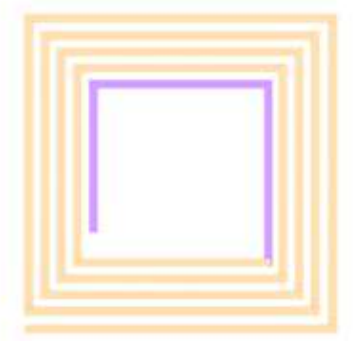

Wireless charging coil

b
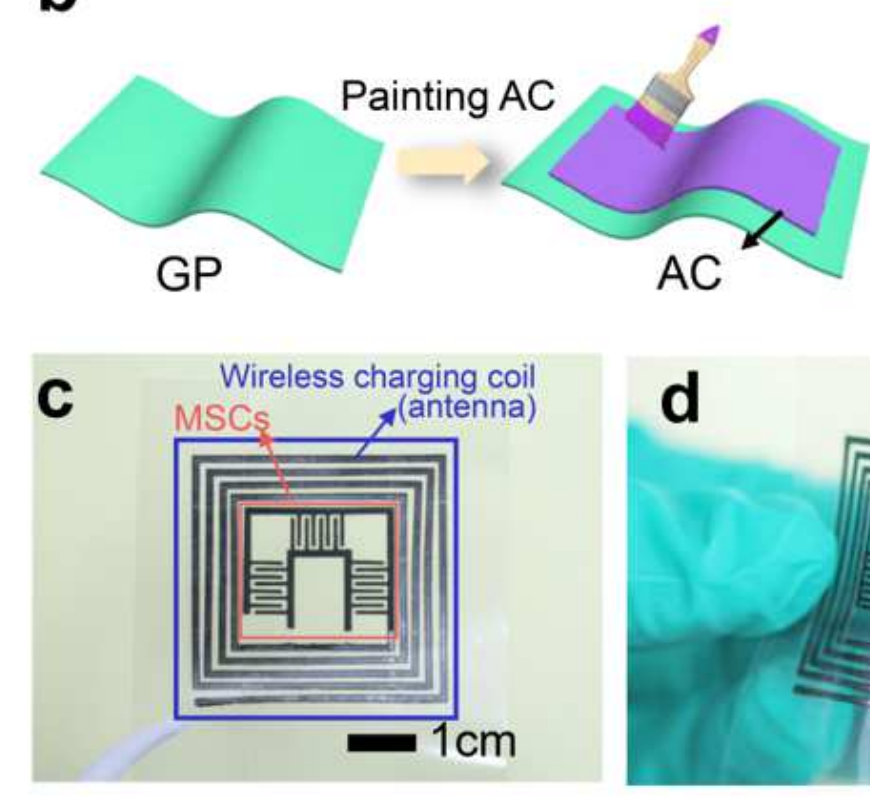

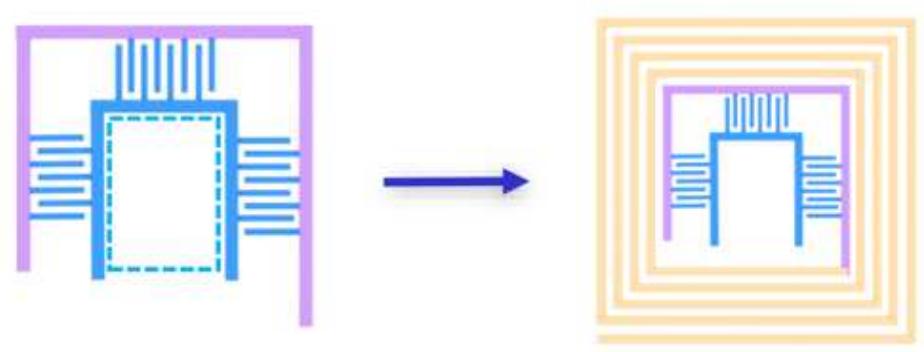

MSCs
Integrated wireless charging MSCs (IWC-MSCs)
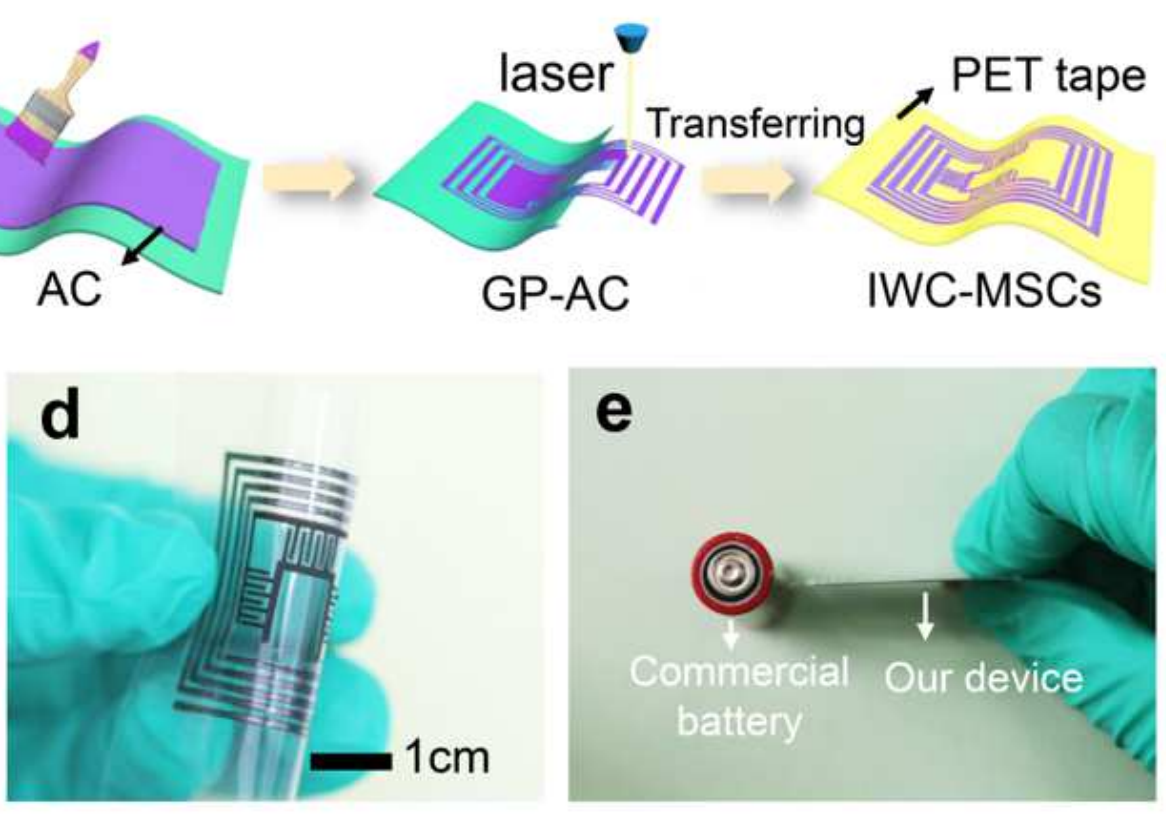

\section{Figure 1}

The design, fabrication and electronic photos of IWC-MSCs. a) The combining of WCC and MSCs in the integrated device, IWC-MSCs. b) The schematic illustration of fabricating the integrated device. c) The image of the integrated device IWC-MSCs. d) Flexibility demonstration of the integrated device IWC-MSCs at a highly folded state. e) Thickness comparison of the commercial battery with IWC-MSCs. 


\section{a}

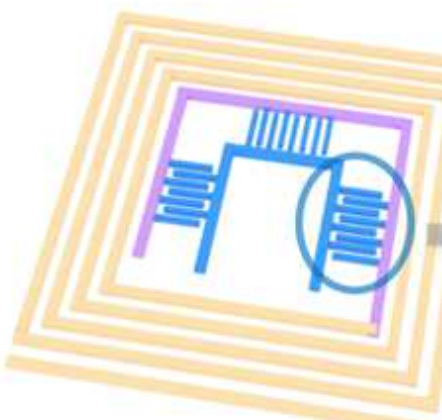

d
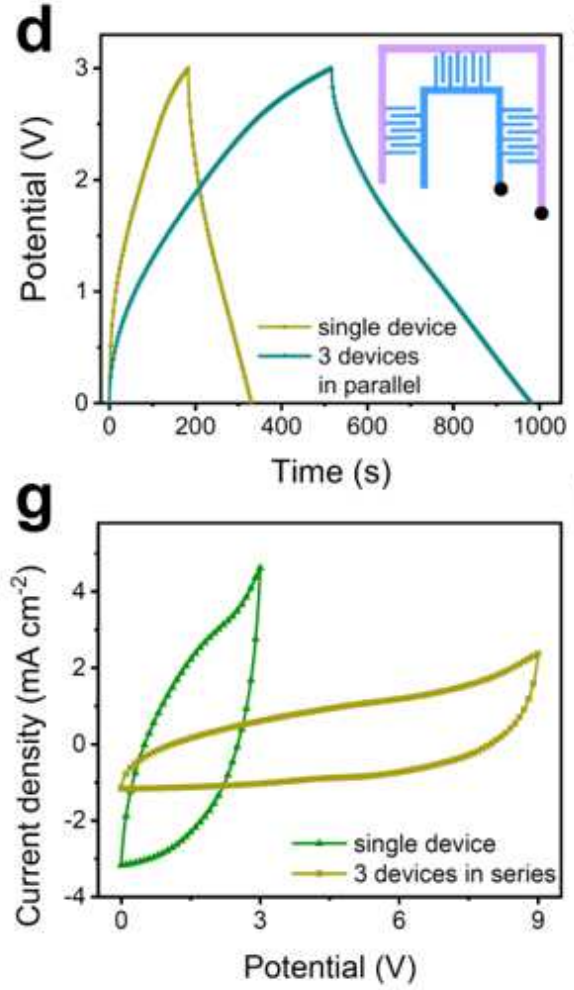

b

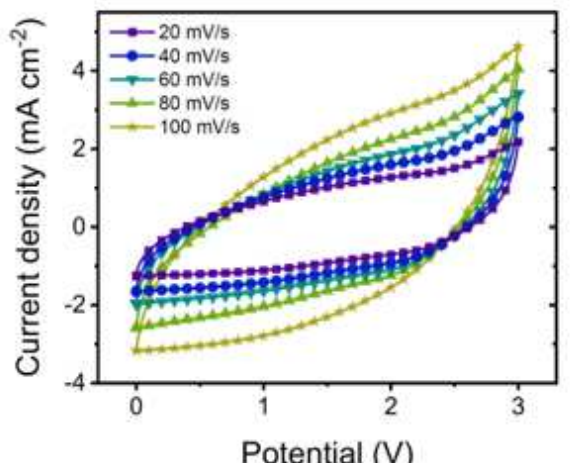

$\mathbf{e}$

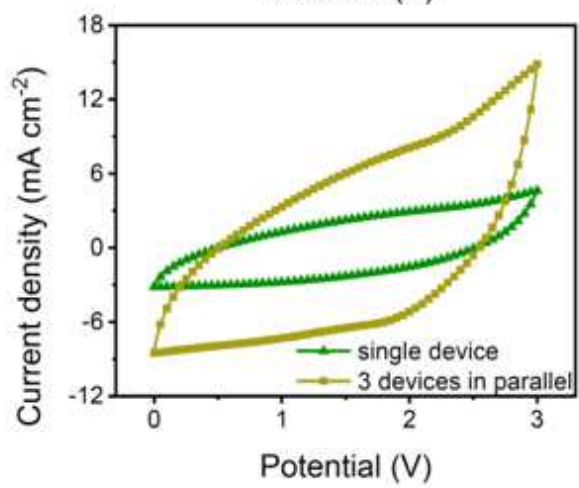

h

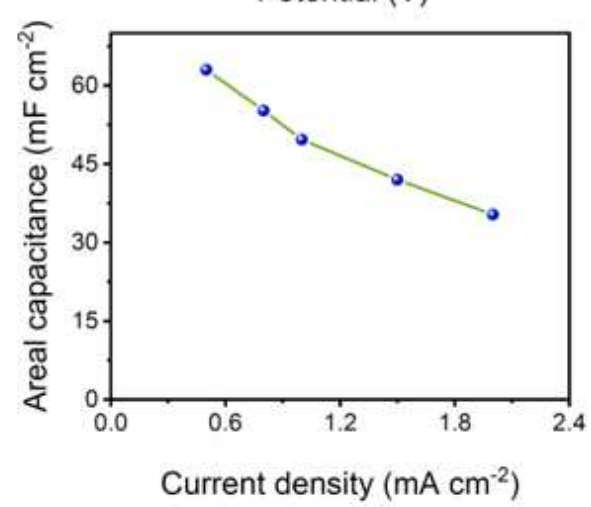

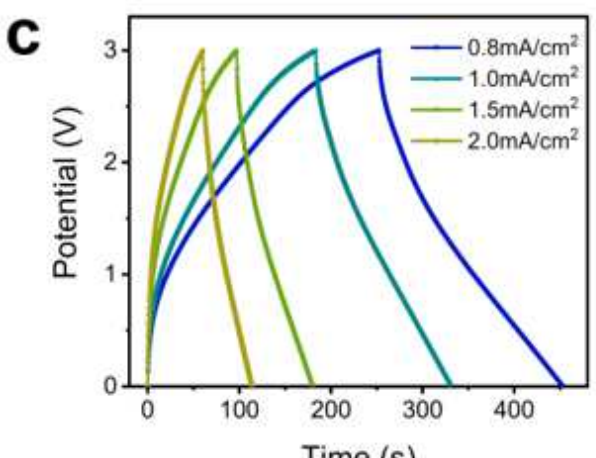

f

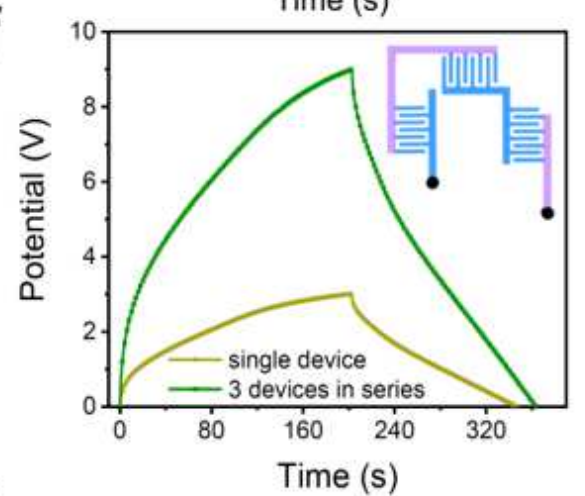

i

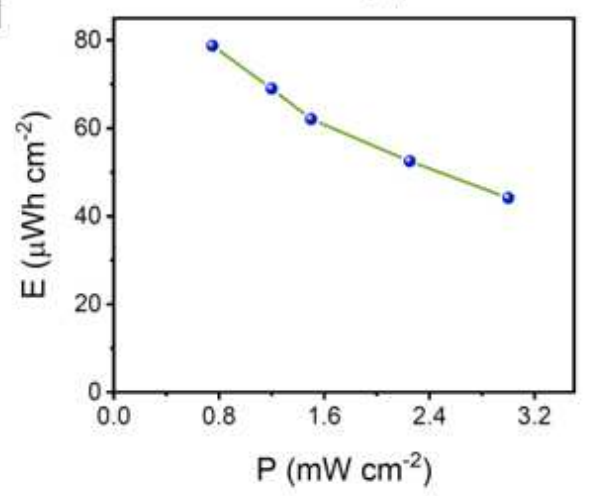

Figure 2

Electrochemical properties of a single MSC in the integrated device. a) The scheme of one MSC in the integrated device. b) CV curves measured at scan rates of 20-100 mV s-1. c) The galvanostatic charging/discharging curves of a single MSC with different current densities. d) Galvanostatic charging/discharging curves of a group of three MSCs connected in parallel. The inset is the schematic diagram of parallel connecting details, where black dots are testing points. e) CV curves of MSCs connected in parallel. f) Galvanostatic charging/discharging curves of a group of three MSCs connected in series. The inset is the schematic diagram of serial connecting details, and the black dots are testing points. g) CV curves of MSCs connected in series. h) The specific capacitance of a single MSC calculated from the discharging time under different currents i) Power and energy densities of a single MSC unit. 


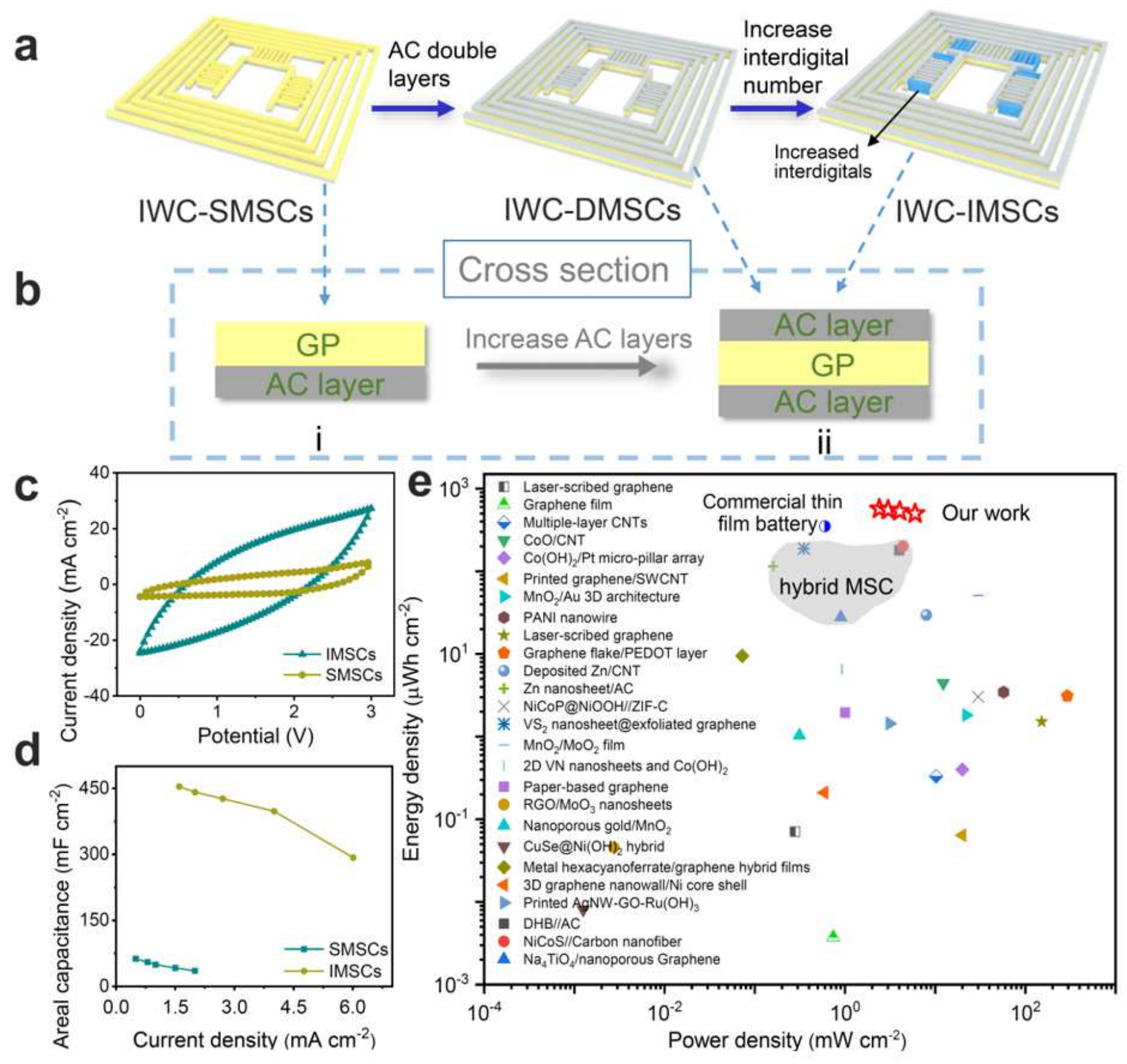

Figure 3

Process and measurements of improving the performance of MSCs in device. a) Schematic diagrams of procedure of optimizing MSCs in IWC-MSCs. b) Change of cross section of SMSCs, DMSCs, and IMSCs. c) Comparison of CV curves under the scanning rate of $100 \mathrm{mV} / \mathrm{s}$ and d) rate capacity curves of MSCs of SMSCs and IMSCs, respectively. e) Ragone plots of the IMSCs compared with other reported MSCs. 


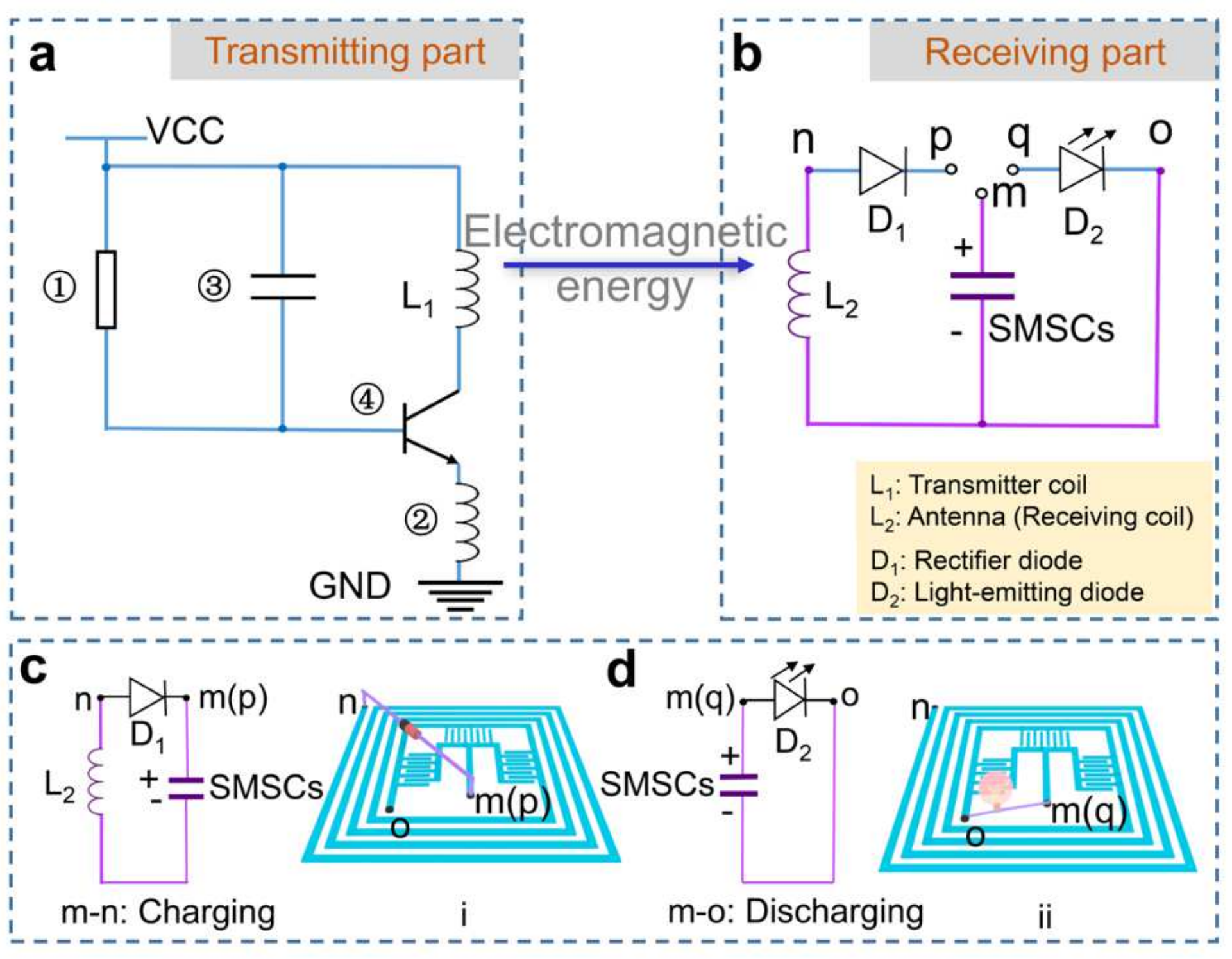

Figure 4

Circuit diagram and working mechanism of wireless charging system. a) Circuit diagram of wireless transmitting part and b) receiving part. c) Schematics of the circuit while connecting point " $\mathrm{m}$ " and " $\mathrm{p}$ " of IWC-SMSCs to charge SMSCs (i). d) Schematics of the circuit while connecting point " $m$ " and " $q$ " of IWCSMSCs to release the accumulated charges in SMSCs (ii), thus lighting a red LED. The IWC-SMSCs is in purple color in the circuit diagrams. 

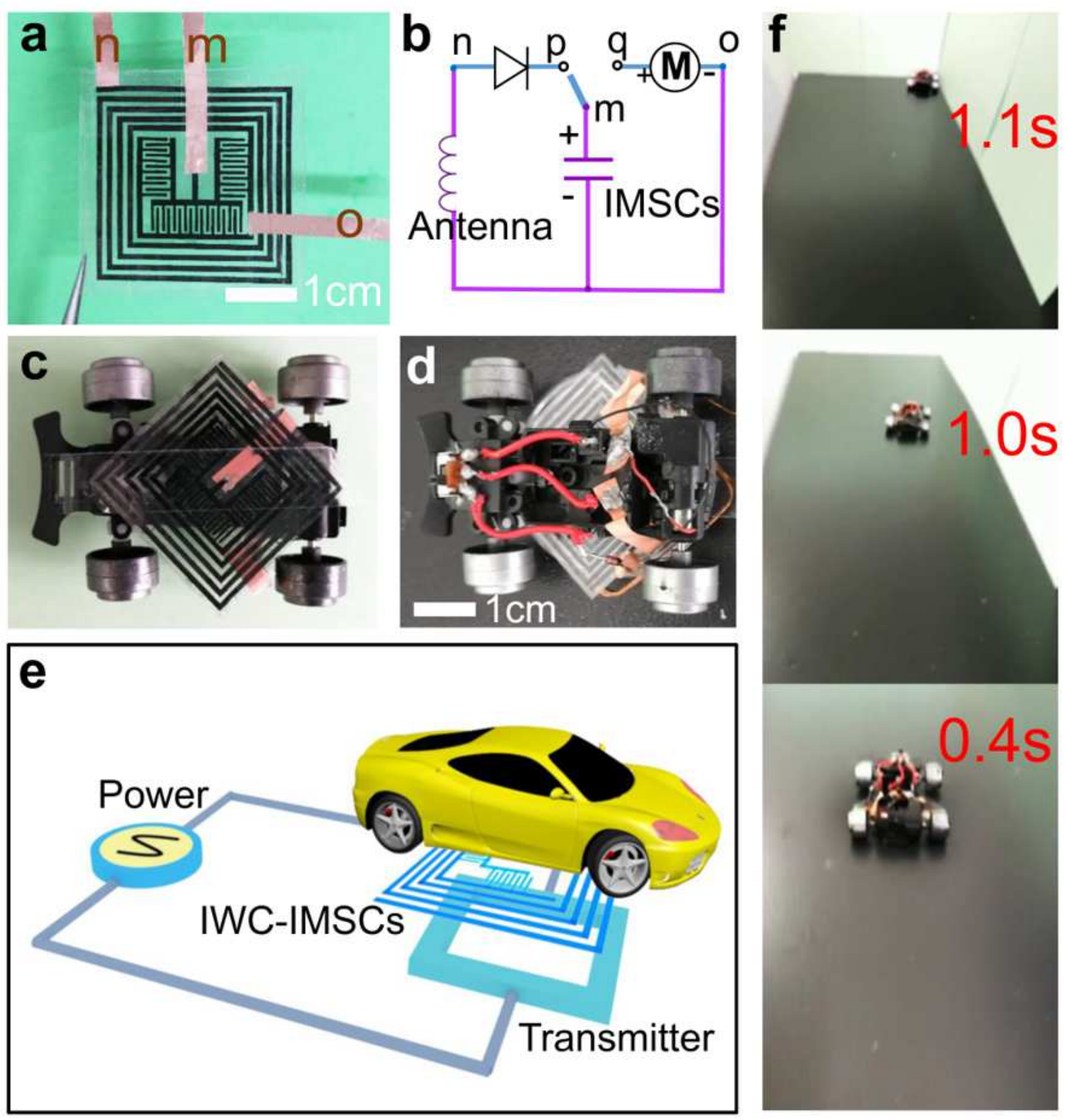

\section{Figure 5}

Assembling IMC-IMSCs with an electrical toy car as a power source. a) An image of the connecting details of IWC-IMSCs using copper foils as conductors. " $m$ ", " $n$ ", "o" copper foils respectively correspond to "m", "n", "o" point in Fig. 5b. b) The circuit diagram of the IWC-IMSCs assembling with the car. c) The downside of the car assembled with IWC-IMSCs. d) The upside of the car connecting with a switch. e) 
The schematic diagram of the wireless charging system. f) The picture of the car with IWC-IMSCs running on the desk.

\section{Supplementary Files}

This is a list of supplementary files associated with this preprint. Click to download.

- SupplementaryMovie1EntirewirelesscharginganddetectingprocesstolightaredLED.avi

- SupplementaryMovie2Anelectricaltoycarstartsrunningafterwirelesscharging.avi

- SupplementaryInformationfinal5.docx 